\title{
Halogen bonds in clathrate cages: A real space perspective
}

José Manuel Guevara-Vela ${ }^{\mathrm{a}}$, David Ochoa-Resendiz ${ }^{\mathrm{b}, \mathrm{c}}$, Ramón Hernández-Lamoneda ${ }^{\mathrm{b}}$, Aurora Costales $^{\mathrm{a}}$, Ángel Martín Pendás ${ }^{\mathrm{a}, *}$

${ }^{a}$ Departament of Analytical and Physical Chemistry, University of Oviedo, E-33006, Oviedo, Spain.

${ }^{b}$ Centro de Investigaciones Químicas, Universidad Autónoma del Estado de Morelos, Av. Universidad 1001, Cuernavaca 62209, Morelos, México.

${ }^{c}$ Instituto Tecnológico de Zacatepec, Calz. Tecnológico 27 Zacatepec 62780, Morelos, México.

\begin{abstract}
In this communication we present real space analyses of the nature of the dihalogen-water cage interactions in the $5^{12}$ and $5^{12} 6^{2}$ clathrate cages containing chlorine and bromine, respectively. Real space analyses, namely the Quantum Theory of Atoms in Molecules and the Interacting Quantum Atoms approach, shown that some interactions in the studied systems present characteristics, such as moderate strenght and electrostatic nature, in agreement with the definition of halogen bonding.
\end{abstract}

Keywords:

Halogen bonding, Interacting quantum atoms, QTAIM, Clathrates

\section{Introduction}

A halogen bond is defined by the IUPAC as a relationship where "...there is evidence of a net attractive interaction between an electrophilic region associated with an halogen atom in a molecular entity an a nucleophilic region in another, or the same, molecular entity" [1]. Even though its full understanding is relatively recent, its relevance is already widely recognized in very different fields, such as biomolecules, crystal engineering, or functional systems design [2].

The nature of guest-host interactions in the case of dihalogen molecules in water clathrate cages ${ }^{1}$ has been the subject of recent debate in both experimental [3? ? ] and theoretical studies $[4 ? ?, 5]$. The initial interpretation of the observed electronic shifts in ultraviolet and visible spectra of bromine clathrate assumed that halogen bonding could not be responsible since all the water lone-pairs are involved in hydrogen bonding of the water lattice and thus not available for halogen bonding [? ]. In contrast the much larger blue-shifts observed in bromine aqueous solutions were consistent with the formation of halogen bonding interactions [? ? ? ? ]. However evidence of halogen bonding in clathrate cages was strongly suggested in ab initio local

${ }^{*}$ To whom correspondence should be addressed: ampendas@uniovi.es

${ }^{1}$ Clathrates are compounds in which the guest molecule is in a cage formed by the host molecule 
correlation calculations through an energy partitioning analysis and calculation of the electronic shifts at selected geometries [5]. This was further confirmed by performing a more thorough calculation of the electronic shifts taking into account the rotational motion of the dihalogen inside the cages [? ]. Another important set of experiments probed the structural characteristics of chlorine and bromine clathrates in X-ray measurements [3]. The case of chlorine has a long and interesting history starting with its accidental discovery by sir Humphrey Davy [? ] in 1811, the first X-ray determination in 1952 by Pauling and Marsh [? ] where using van der Waals radii arguments it was assumed that chlorine would not fit in the smaller dodecahedral cage and the recent detailed study by the group of Ripmeester showing that more than $30 \%$ of the $5^{12}$ dodecahedral cages are occupied [3]. Furthermore in the case of the mixed clorine and bromine clathrate a strong distortion of the $5^{12}$ cage and a short $\mathrm{Cl}$-O distance $(2.7 \AA)$ together with lengthening of the nearby hydrogen bonds could be an indication of the presence of halogen bonding interactions.

Previous theoretical analyses have focused so far on orbital based energetic partitionings that do not allow for discriminating directly halogen bonds from other types of interactions. In the meantime, a considerable amount of work has shown that spatial features of the electron density $\rho$, like the position of charge concentration (CC) and charge depletion (CD) regions of the Laplacian of the density, $\nabla^{2} \rho$, lead to unambiguous descriptors in these systems. For instance, Espinosa and coworkers[? ] have shown that halogen bonded synthons, used in crystal engineering, are characterized by a clear match between CC's and CD's that can be interpreted electrostatically, in agreement with the $\sigma$-hole paradigm. We are not aware that any of these techniques have been used to date to establish the nature of the bonding between dihalogens and clathrate cages. We hereby use a battery of methods, that range from the description of the topological features of the electron density in these systems as provided by the quantum theory of atoms in molecules (QTAIM), passing through an orbital free energetic decomposition based on QTAIM basins, the so-called interacting quantum atoms approach (IQA), and including arguments borrowed from the analysis of the electron localization function (ELF) and the noncovalent interaction index (NCI). As we will show, our results provide strong indications that halogen bonding is present even though the water molecules lone pairs are already engaged into hydrogen bonding interactions.

\section{Methodology}

We have considered a combination of real space analyses that could further advance the understanding of the guest-host relation. The Quantum Theory of Atoms in Molecules (QTAIM), 
based on the structure of the electron density scalar field, is an appropiate technique in the research of intra-atomic interactions, providing a separation of the molecular real space into disjoint regions. The latter, constructed as the attraction basins of the density, are identified with quantum atoms. All standard quantum-mechanical observables in IQA can be decomposed into intra- and (at most) interatomic contributions exactly. [6].

The QTAIM has been extensively used for investigations in diverse problems related with intra- and intermolecular interactions. We can cite, for instance, the nature of endohedral complexes [7, 8], hydrogen bonds [9-11] or photoisomerization [12] processes, the anomeric effect [13], etc. In this regard, halogen bonding is not unknown to these analyses, having been widely explored by Grabowski,[14] or by Espinosa and coworkers as crystal engineering is regarded.[? ] A real space, thus orbital invariant, decomposition of the molecular energy compatible with the QTAIM partitioning is provided by the Interacting Quantum Atoms (IQA) approach.[15, 16] IQA writes the total molecular energy as a sum of intra- and interatomic terms that gauge the interaction between atomic domains:

$$
E=\sum_{\mathrm{A}} E_{\text {net }}^{\mathrm{A}}+\sum_{\mathrm{A}>\mathrm{B}} E_{\text {int }}^{\mathrm{AB}}
$$

where $E_{\text {net }}^{\mathrm{A}}$ is the net energy of atom $\mathrm{A}$, which contains the kinetic and potential energy terms of all particles contained in domain $\mathrm{A}$, and $E_{\mathrm{int}}^{\mathrm{AB}}$ is the interaction energy between atoms $\mathrm{A}$ and $\mathrm{B}$ that gathers all the inter-particle potential energy contributions of particles in domain A with particles in domain B. The interaction energy between two basins can be further separated into a classical component, which is assimilated as the ionic contribution to the interaction $V_{\mathrm{cl}}^{\mathrm{AB}}$ and an exchange correlation one, $V_{\mathrm{xc}}^{\mathrm{AB}}$, which measures the covalent term.

$$
E_{\mathrm{int}}^{\mathrm{AB}}=V_{\mathrm{cl}}^{\mathrm{AB}}+V_{\mathrm{xc}}^{\mathrm{AB}}
$$

Because of this distinctive ability of separating bonding components as well as reconstructing fully the energy in a pairwise additive manner, IQA has been succesfully employed to scrutinize a variety of phenomena where there is a combination of Coulombic and quantum contributions, like the cooperativity in hydrogen bonds $[17,18]$, the role of covalency in metal-metal interactions [19] or in halogen bonding [20-22].

Since lone pairs are an essential part of the accepted $\sigma$-hole paradigm that is usually employed to describe halogen bonds, we have also used real space techniques which stress the role of these objects. The laplacian of the density, $\nabla^{2} \rho$, a field that recovers the atomic shell structure and its symmetry breaking in molecules, has been traditionally used to uncover the position of 
those objects. However, it is well known that the outer shells are not captured by $\nabla^{2} \rho$ beyond $Z=20$ (the calcium atom). Since we are not only dealing with $\mathrm{Cl}(Z=17)$, but also with $\mathrm{Br}$ $(Z=35)$, we have decided to examine the electron localization function (ELF) of Becke and Edgecombe,[23] which was further popularized in chemistry by Savin and Silvi [24]. This scalar, which can be interpreted as measuring the excess kinetic energy density of an electron system with respect to the bosonic von Weizsäcker reference, provides stunning images of great chemical value by, grossly speaking, locating regions where electrons are localized. It also provides an exhaustive partition of space into regions that are now associated to cores, lone pairs, and bonds instead of atoms. As we will show, ELF provides important insights about the characterization of lone pairs and $\sigma$-holes, both essential elements in halogen bonding.

Similarly, contemporary research is continuously emphasizing the role that dispersion forces play in building the overall architecture of molecular aggregates. Given the rather delocalized nature of these interactions, the standard local QTAIM analysis based on descriptors obtained at critical points is not particularly suited to uncover the importance of these forces for a given interaction. To this end, the non covalent interactions index (NCI) introduced by Johnson and coworkers [? ] has shown to be particularly useful. The NCI index is also a kinetic energy based descriptor, this time using the reduced density gradient $s(\boldsymbol{r}) \approx|\nabla \rho(\boldsymbol{r})| / \rho(\boldsymbol{r})^{4 / 3}$. In intermolecular regions, minima of $s$ uncover non-covalent interactions, that can be further classified if the second eigenvalue of the hessian of the electron density $\left(\lambda_{2}\right)$ is also used. Isosurfaces of the NCI index rainbow-coloured through $\lambda_{2}$, with blue and red corresponding to (very) negative and (very) positive $\lambda_{2}$ values, respectively, provide very intuitive images of intermolecular interactions. Strongly blue isosurfaces are found, for instance, in hydrogen bonds, while strongly red ones tend to uncover sterically crowded regions. The NCI picture of the dihalogen clathrates adds to the global QTAIM, IQA, and ELF descriptions.

\section{Computational Details}

We have specifically studied $\mathrm{Cl}_{2}$ and $\mathrm{Br}_{2}$ molecules confined in the $5^{12}$ and the $5^{12} 6^{2}$ clathrate cages, respectively. A plot of both systems is shown in Figure 1.

The geometries for the clathrate complexes have been taken from those reported in a previous local correlation study [5] where evidence of halogen bonding was found for the $\mathrm{Cl}_{2} @ 5^{12}$ cage in the global energy minimum but not in $\mathrm{Br}_{2} @ 5^{12} 6^{12}$, where it appeared at a geometry which does not correspond to a total energy minimum. Using the local correlation energy partitioning analysis in this case, it was possible to establish a clear correlation with the ionic energy component and for this reason the geometry at which this component is minimum is also presented 

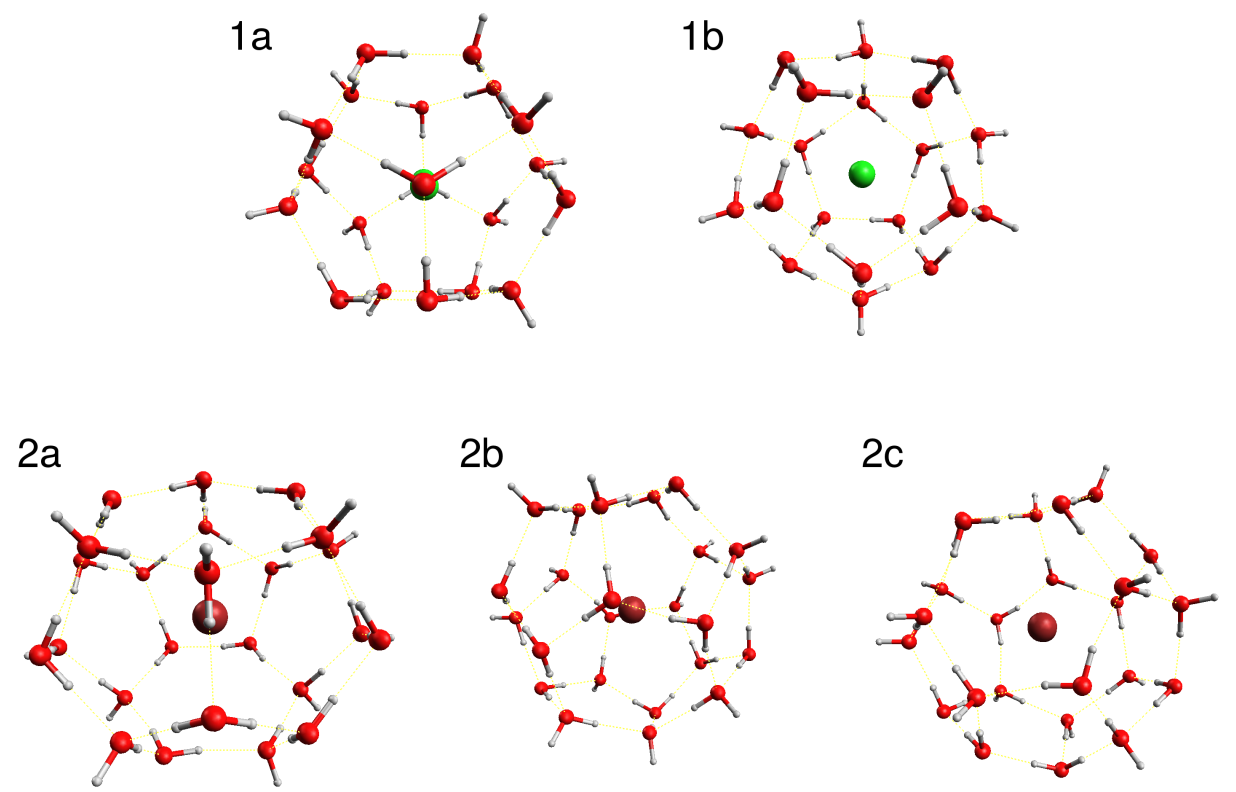

Figure 1: Sketch of the systems studied in this work. $\mathrm{Cl}_{2}$ in the $5^{12}$ cage at the global minimum (1a) and maximum (1b) conformations, and $\mathrm{Br}_{2}$ in the $5^{12} 6^{2}$ cage in three conformations: the global minimum (2a), the conformation at which the ionic energetic component is minimum (2c, see the text for further information), and the global maximum (2c).

and discussed.

The electronic structure calculations necessary to obtain the density were done at the M06X/aug-cc-pVTZ [26, 27] level of theory using the GAUSSIAN09 package. [28] The QTAIM and IQA analyses were carried out with the AIMAll program [29] while the ELF calculations were made using our in house PROMOLDEN code [30]. The IQA computations was done employing an approximately reconstructed second order reduced density matrix [31].

\section{Results and discussion}

\subsection{QTAIM topology}

A local QTAIM analysis reveals that in both complexes, regardless of their specific conformation, numerous bond paths exist that link the halogen atoms to the oxygens in the clathrate cage, as shown in Figure 2. Besides being traditionally associated with bonds in simple chemical compounds [6], in more controvertial or complex situations bond paths are indicators of preferred quantum-mechanical exchange channels between atoms.[32] Their presence here is a first sign of non-negligible interactions between the halogen atoms and some of the oxygens forming the water molecules in the cage. 

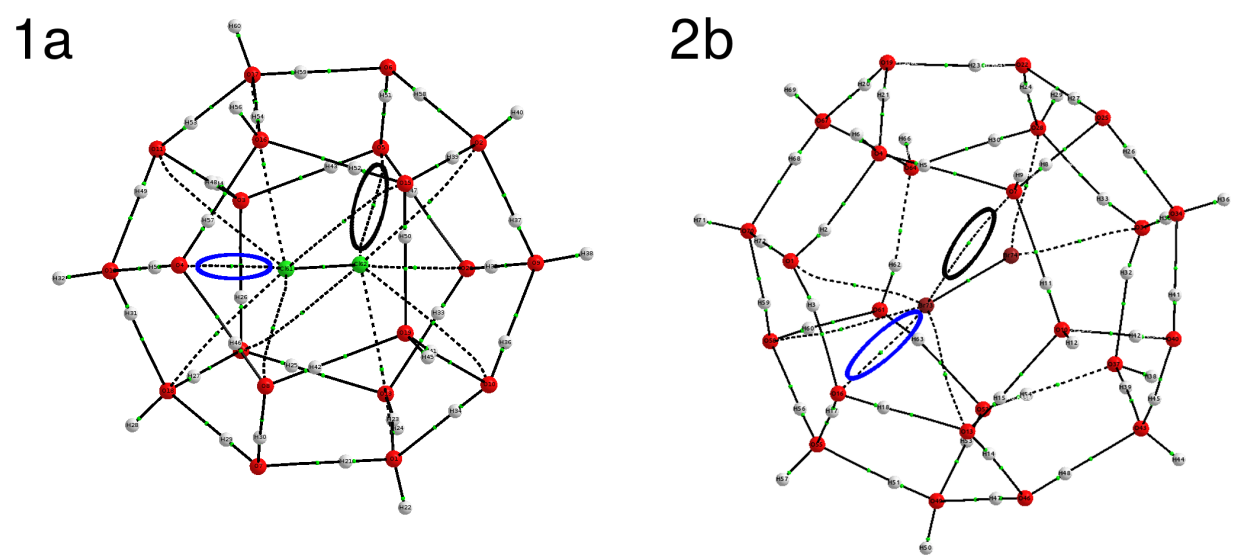

Figure 2: QTAIM bond paths for systems $1 \mathrm{a}$ and 2b. In blue we highlight two oxygen-halogen interactions that are (semi) colinear to the halogen-halogen bond (Cl61-O4 and Br73-O16). Similarly, two representants of nonlinear interactions are indicated in black (Cl62-O5 and Br73-O7).

However, not all the bond paths present in the different conformations should be thought of as equivalent. Actually, their distances and angles with respect to the halogen-halogen axis are very different. On the one hand we have those interactions that fulfil the condition of linearity needed for being considered as halogen bonds, and on the other hand we can gather those that do not, i.e., those that form a considerable angle or are even perpendicular to the halogenhalogen axis. This is shown in Figure 2, where two of the former are highlighted in blue while two of the latter are pointed out in black.

Table 1 summarizes the geometrical parameters as well as several bond critical point (BCP) QTAIM descriptors of the interactions that are more likely to be considered as halogen bonds, the Cl61-O4 bond path in 1a and the Br73-O16 one in 2b, together with other oxygen-halogen contacts selected for comparison, and the reference systems $\mathrm{Cl}_{2} \cdots \mathrm{H}_{2} \mathrm{O}$ and $\mathrm{Br}_{2} \cdots \mathrm{H}_{2} \mathrm{O}$.

As the local QTAIM indicators at BCPs are regarded, the density at the bond critical point in these compounds is closely related to the distance between the atoms involved, and thus offers little extra information related to the nature of the interaction. The Laplacian of the density $\left(\nabla^{2} \rho\right)$, traditionally used to separated closed-shell (if positive) from shared-shell (if negative) behavior, reproduces this trend, magnifying it. In all of the cases, its small positive value implies weak closed-shell interactions, as expected. All in all, these simple local descriptors do neither confirm nor deny the halogen bond nature of the guest-host bonding. More information can be obtained from the analysis of other scalar fields. 
Table 1: Geometric parameters and several local QTAIM properties of some bond paths present in the different conformations examined. $\rho_{b}$ and $\nabla^{2} \rho_{b}$ refer to the values of the electron density and its laplacian at the bond critical point.

\begin{tabular}{llcccc}
\hline System & Interaction & Distance $/ \AA$ & $\angle$ OXX/degree & $\rho_{b} /$ a.u. & $\nabla^{2} \rho_{b} /$ a.u. \\
\hline $\mathrm{Cl}_{2}-\mathrm{H}_{2} \mathrm{O}$ & Cl2-O3 & 2.771 & 180.0 & 0.0157 & 0.0698 \\
$1 \mathrm{a}$ & Cl61-O4 & 2.790 & 175.2 & 0.0174 & 0.0731 \\
& Cl62-O5 & 3.204 & 130.3 & 0.0096 & 0.0348 \\
$\mathrm{1b}$ & $\mathrm{Cl61-O18}$ & 2.835 & 133.9 & 0.0153 & 0.0696 \\
\hline $\mathrm{Br}_{2}-\mathrm{H}_{2} \mathrm{O}$ & $\mathrm{Br2-O} 3$ & 2.796 & 179.5 & 0.0181 & 0.0732 \\
$2 \mathrm{a}$ & $\mathrm{Br74-O} 7$ & 3.159 & 165.1 & 0.0081 & 0.0334 \\
$2 \mathrm{~b}$ & Br73-O7 & 4.237 & 84.2 & 0.0014 & 0.0047 \\
& Br73-O16 & 2.788 & 167.0 & 0.0209 & 0.0803 \\
& Br74-O31 & 2.895 & 162.4 & 0.0176 & 0.0705 \\
2c & Br73-O31 & 3.044 & 141.2 & 0.0146 & 0.0548 \\
\hline
\end{tabular}

\subsection{ELF isosurfaces and the role of lone pairs}

The importance of the ELF analysis for this study thrives due to its ability to bring into focus the presence of lone pairs. The standard electrostatic model of halogen bonding rests heavily on the geometrical disposition of lone pairs. In typical cases it is the presence of a $\sigma$-hole in the halogen, accompanied by a lone pair in the partner that acts as a Lewis base, that is used to recognize the interaction as a halogen bond. Here, the purported saturation of all the lone pairs of the oxygen atoms belonging to the water molecules of the cage, which are engaged in normal hydrogen bonds, forms the basis for denying the presence of halogen bonds.

Fig. 3 shows relevant ( $\eta=0.9$ a.u) ElF isosurfaces. It is first interesting to recognize the archetypal halogen bond pattern in the $\mathrm{Cl}_{2} \cdots \mathrm{H}_{2} \mathrm{O}$ and $\mathrm{Br}_{2} \cdots \mathrm{H}_{2} \mathrm{O}$ complexes. In both cases, the halogen's lone pairs adopt a quasi-cyllindrical arrangement seen in the ELF as a well developed localization torus around the halogen atom. This leaves a region of much smaller $\eta$ values that can be put in correspondence with the electrostatic potential sigma-hole. Clearly, one of the two lone pairs of the oxygen atoms points directly toward the $\sigma$-hole. This view is in agreement with a donor-acceptor paradigm, and explains the lock-and-key geometry which is observed. Notice that more subtle effects can also be recognized from a more detailed analysis of the ELF function. For instance, it is clear that the two lone pairs of the donor water molecule cease to be equivalent in the complex, and that some electronic charge is transferred toward the lone pair pointing away from the dihalogen in an attempt to minimize interelectron repulsions. 

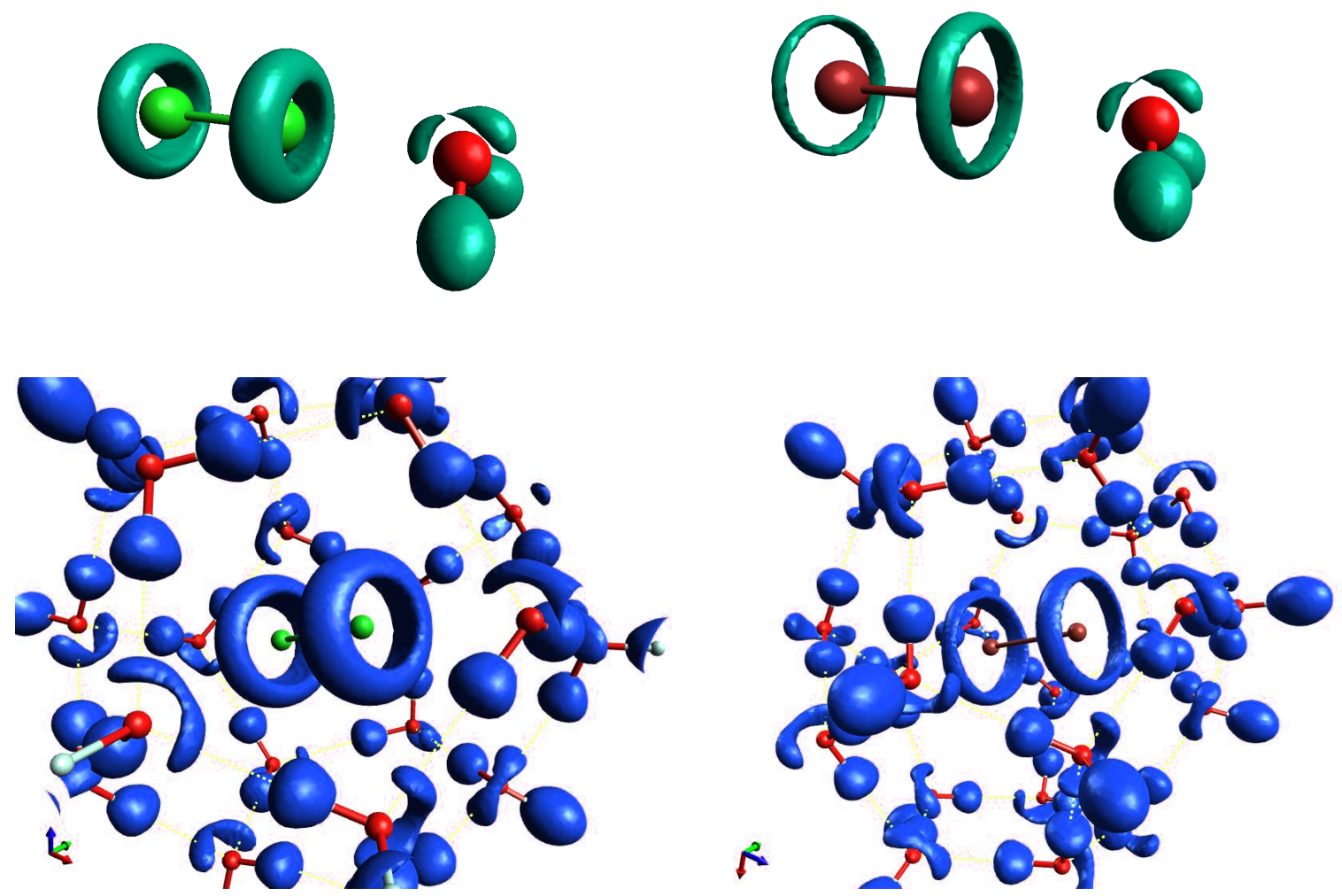

Figure 3: $\eta=0.9$ ELF isosurfaces for the $\mathrm{Cl}_{2} \cdots \mathrm{H}_{2} \mathrm{O}$ and $\mathrm{Br}_{2} \cdots \mathrm{H}_{2} \mathrm{O}$ complexes (top, from left to right), and for systems $1 \mathrm{a}$ and $2 \mathrm{~b}$ (bottom, also from left to right). The geometry of both the $\sigma$-holes and the oxygens' lone pairs is highlighted. 
The ELF isosurfaces of clathrates $1 \mathrm{a}$ and $2 \mathrm{~b}$ show that although the lone pairs of the oxygen atoms of the cage are basically engaged in hydrogen bonds in the expected $\mathrm{H}_{2} \mathrm{O}(\mathrm{LP})$...H-OH manner, the closest X $\cdots \mathrm{O}$ contacts involve a lock-and-key distribution of part of a lone pair

and the X sigma-hole. This lone pair is both participating in a hydrogen bond and in what we will call a partial halogen bond. Notice that these contacts are the same appearing in Tab. 1 as those with largest densities and laplacians and smallest X-O distances. Moreover, the rest of the oxygens' lone pairs point either outside the clathrate cage or lie vaguely around the halogen's tori, disqualifying these contacts as halogen bonds.

\subsection{NCI isosurfaces and the delocalized nature of the $X_{2}$ cage interactions}

Figure 4 shows the NCI equivalent to Fig. 3. It is first interesting to show that the reference $\mathrm{Cl}_{2} \cdots \mathrm{H}_{2} \mathrm{O}$ and $\mathrm{Br}_{2} \cdots \mathrm{H}_{2} \mathrm{O}$ complexes develop a rather standard, blue disk-shaped surface between the halogen and the oxygen atom, in complete agreement with the ELF picture that points toward the importance of lone pairs. In the $1 \mathrm{a}$ and $2 \mathrm{~b}$ clathrates the overall image is confirmed. The figure emphasizes how all the water-water hydrogen bonds are well developed, and how this does not preclude the appearance of rather intense blue contact surfaces between the chlorine or bromine atoms and the closest oxygen atoms, again in agreement with the lock and key arrangement found in the ELF. As expected, the NCI index shows that the interaction between the dihalogen and the cage involves a considerable number of the atoms of the cage (notice the extended nature of the isosurfaces), but it is also clear that the most attractive ones are those that have been previously identified as potential halogen bonds.

In this sense, both the ELF and NCI descriptions provide compatible interpretations favouring the existence of halogen bonding in the $1 \mathrm{a}$ and $2 \mathrm{~b}$ conformers.

\subsection{IQA interaction energies}

Although both a standard QTAIM analysis at BCPs as well as the chemical images provided by the ELF and NCI agree with a non-negligible role of halogen bonding in the stabilization of the $1 \mathrm{a}$ and $2 \mathrm{~b}$ clathrates, a more convincing argument coming from energetic descriptors can also be put forward. To that end we have used the IQA approach to dissect the features of individual interatomic interactions, specifically as their relative strength with respect to the $\mathrm{X}_{2} \cdots \mathrm{H}_{2} \mathrm{O}$ systems are regarded. A summary of our results can be found in Tab. 2.

It is firstly noteworthy that in the isolated $\mathrm{X}_{2} \cdots \mathrm{H}_{2} \mathrm{O}$ complexes, the direct halogen-oxygen IQA interaction energy is non-negligible, about -24 and $-34 \mathrm{kcal} / \mathrm{mol}$ in the chlorine and bromine compounds. Even more interesting is noticing that both the electrostatic (classical) and covalent (exchange-correlation) contributions are important. $V_{\mathrm{cl}}$ is slightly more relevant 
a)

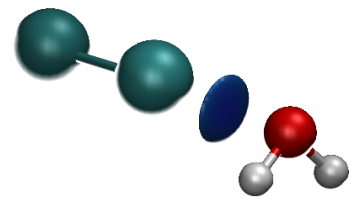

c)

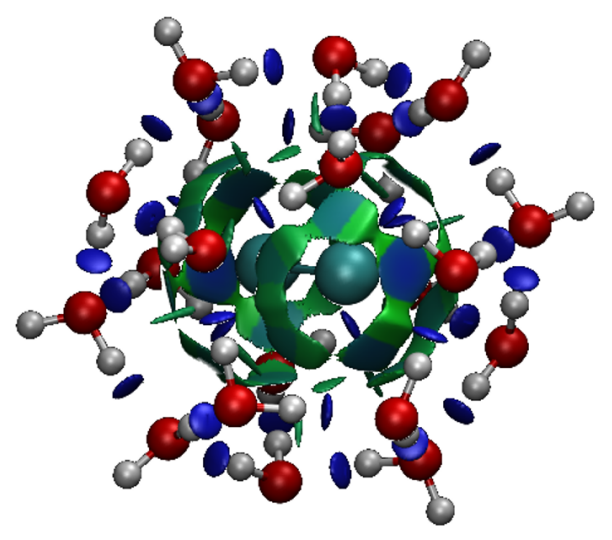

b)

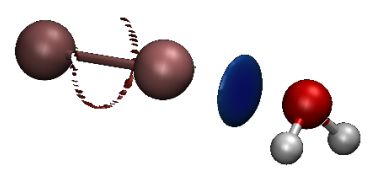

d)

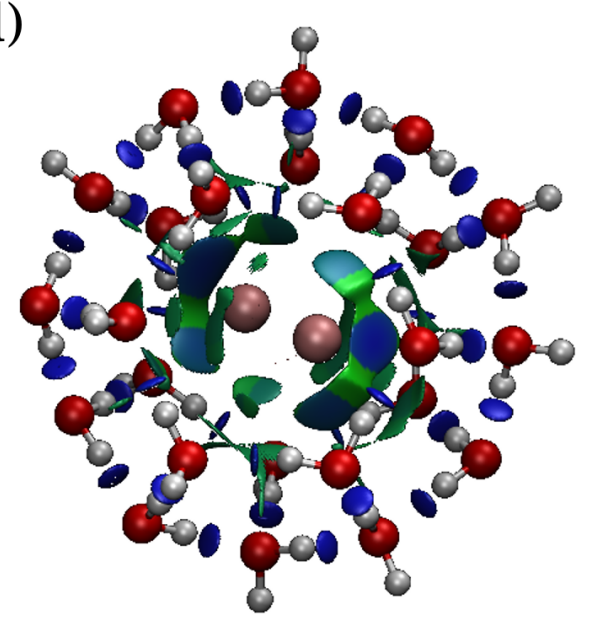

Figure 4: NCI isosurfaces for the $\mathrm{Cl}_{2} \cdots \mathrm{H}_{2} \mathrm{O}$ and $\mathrm{Br}_{2} \cdots \mathrm{H}_{2} \mathrm{O}$ complexes (top, from left to right), and for systems $1 \mathrm{a}$ and $2 \mathrm{~b}$ (bottom, also from left to right).

in $\mathrm{Br}_{2} \cdots \mathrm{H}_{2} \mathrm{O}$, in agreement with the increased intensity of the $\sigma$-hole in this case ESTAS DE ACUERDO, RAMON?. The importance of $V_{\mathrm{xc}}$ is in line with previous IQA work on hydrogen bonding[? ]. In more traditional molecular orbital descriptions, this can be read as an important overlap contribution. When other factors are equal, the exchange-correlation term of a given weak interaction is then mostly dependent on the distance between the atoms involved. Thus, although $V_{\mathrm{xc}}$ is essential for the final energetic stabilization of the complex, in a sense the IQA marker of the presence of a halogen bond is a strong and attractive $V_{\mathrm{cl}}^{\mathrm{AB}}$.

Although not surprising to the electrostatic potential connoisseur, the stabilizing (negative) values of $V_{\mathrm{cl}}$ reported in the Table require further explanation, since both the halogen atoms and the corresponding oxygens bear a negative QTAIM net charge. Since the monopole-monopole electrostatic contribution usually dominates, a negative $V_{\mathrm{cl}}$ can only be the result of large counteracting dipole and quadrupole terms, i.e. of considerable density polarizations. These can be pictorially attributed to the lone pair $/ \sigma$-hole interaction. A corroboration is found for the Cl62-O5 atomic pair in the 1a conformer, where the classical part of the interaction energy is sligthly positive as in this case the interaction occurs between an oxygen's lone pair and the lone pairs of the adjacent chlorine atom.

As the clathrate cages are formed and the water molecules engage in hydrogen bonding, the 
Table 2: Relevant IQA descriptors between selected oxygen-halogen interactions. All energies in kcal/mol.

\begin{tabular}{|c|c|c|c|c|}
\hline System & Interaction & $E_{\mathrm{int}}^{\mathrm{AB}}$ & $V_{\mathrm{cl}}^{\mathrm{AB}}$ & $V_{\mathrm{xc}}^{\mathrm{AB}}$ \\
\hline $\mathrm{Cl}_{2} \cdots \mathrm{H}_{2} \mathrm{O}$ & $\mathrm{Cl} 2-\mathrm{O} 3$ & -24.48 & -10.36 & -14.12 \\
\hline \multirow[t]{2}{*}{$1 \mathrm{a}$} & Cl61-O4 & -21.74 & -7.20 & -14.54 \\
\hline & Cl62-O5 & -5.61 & 0.63 & -6.25 \\
\hline $1 b$ & Cl61-O18 & -12.12 & -1.60 & -10.52 \\
\hline $\mathrm{Br}_{2} \cdots \mathrm{H}_{2} \mathrm{O}$ & Br2-O3 & -34.07 & -17.06 & -17.01 \\
\hline $2 \mathrm{a}$ & B & -13 & -7.02 & -6.18 \\
\hline \multirow[t]{3}{*}{$2 b$} & $\mathrm{Br} 73-\mathrm{O} 7$ & -2.06 & -1.35 & -0.70 \\
\hline & Br73-O16 & -37.78 & -19.49 & -18.29 \\
\hline & Br74-O31 & -19.61 & -5.36 & -14.24 \\
\hline $2 \mathrm{c}$ & Br73-O31 & -14.62 & -3.61 & -11.01 \\
\hline
\end{tabular}

closest halogen-oxygen interactions are modified. As the data displayed in the Table shows, this new environment decreases the Cl61-O4 $E_{\text {int }}$ in the $\mathrm{Cl} 5^{12}$ cage to about $-22 \mathrm{kcal} / \mathrm{mol}$. This $2.7 \mathrm{kcal} / \mathrm{mol}$ destabilization is basically due to $V_{c l}$, and is probably associated to a non-ideal geometry with respect to the $\mathrm{Cl}_{2} \cdots \mathrm{H}_{2} \mathrm{O}$ case. The Cl62-O5 pair, with a larger $\mathrm{Cl}-\mathrm{O}$ distance and a non-aligned $\sigma$-hole, oxygen pair results in a slightly positive $V_{c l}$, as already commented, and a much smaller exchange contribution. If we restrict to the Cl61-O4 pair, the IQA energetic description fully agrees with a $\mathrm{Cl} \cdots \mathrm{O}$ halogen bond, slightly weaker than that in the reference complex.

Similar conclusions can be drawn from the bromine cages. Since the Br73-O16 pair has a slightly smaller $\mathrm{Br}-\mathrm{O}$ distance than that found in the isolated $\mathrm{Br}_{2} \cdots \mathrm{H}_{2} \mathrm{O}$ complex, both the electrostatic and exchange components of the interaction increase slightly, $V_{\mathrm{cl}}$ feeling a slightly stronger effect. Overall, the $\mathrm{Br}-\mathrm{O} V_{\mathrm{cl}}$ term almost doubles its chlorine counterpart. The large interaction energy is again the result of combining a larger $\sigma$-hole for the bromine atom, and a more flexible geometry afforded by a bigger clathrate cage. Again, the Br73-O16 interaction cannot and should not be distinguished from the widely accepted halogen bonding found in $\mathrm{Br}_{2} \cdots \mathrm{H}_{2} \mathrm{O}$.

\section{Conclusions}

Real space analyses of the nature of the dihalogen-water cage interactions in the $5^{12}$ and $5^{12} 6^{2}$ clathrate cages containgin chlorine and bromine, respectively, show that indeed the QTAIM 
topological characteristics of the studied systems are indicative of the presence of halogen bonding. Moreover, the energies associated to the proposed halogen bonds computed by IQA are comparable to those corresponding to the model systems $\mathrm{H}_{2} \mathrm{O} \cdots \mathrm{Cl}_{2}$ and $\mathrm{H}_{2} \mathrm{O} \cdots \mathrm{Br}_{2}$. Our results are in agreement with the vision of this interactions as halogen bonds.

\section{References}

[1] G. R. Desiraju, P. S. Ho, L. Kloo, A. C. Legon, R. Marquardt, P. Metrangolo, P. Politzer, G. Resnati, K. Rissanen, Pure and Applied Chemistry 2013, 85, 1711-1713.

[2] G. Cavallo, P. Metrangolo, R. Milani, T. Pilati, A. Priimagi, G. Resnati, G. Terraneo, Chem. Rev. 2016, $116,2478-2601$.

[3] K. A. Udachin, S. Alavi, J. A. Ripmeester, J. Phys. Chem. C 2013, 117, 14176-14182.

[4] R. Franklin-Mergarejo, J. Rubayo-Soneira, N. Halberstadt, K. C. Janda, V. A. Apkarian, J. Chem. Phys. 2016, $144,054307$.

[5] D. Ochoa-Resendiz, F. A. Batista-Romero, R. Hern??ndez-Lamoneda, J. Chem. Phys. 2016, 145.

[6] R. F. W. Bader, Atoms in molecules: A Quantum Theory, Oxford University Press, 1990.

[7] A. a. Popov, S. M. Avdoshenko, A. M. Pendás, L. Dunsch, Chem. Commun. 2012, 48, 8031.

[8] J. M. Guevara-Vela, T. Rocha-Rinza, Á. M. Pendás, Theor. Chem. Acc. 2017, 136, 1-5.

[9] T. Cristina, G. Sánchez-Sanz, I. Alkorta, J. Elguero, O. Mó, M. Yáñez, J. Mol. Struct. 2013, 1048, 138-151.

[10] J. M. Guevara-Vela, E. Romero-Montalvo, A. Costales, A. M. Pendas, T. Rocha-Rinza, Phys. Chem. Chem. Phys. 2016, 18, 26383-26390.

[11] E. Romero-Montalvo, J. M. Guevara-Vela, A. Costales, Á. Martín Pendás, T. Rocha-Rinza, Phys. Chem. Chem. Phys. 2017, 19, 97-107.

[12] L. Gutiérrez-Arzaluz, T. Rocha-Rinza, F. Cortés-Guzmán, Comp. Theor. Chem. 2015, 1053, 214-219.

[13] D. Ferro-Costas, R. a. Mosquera, J. Chem. Theory Comput. 2013, 9, 4816-4824.

[14] S. J. Grabowski, J. Phys. Chem. A 2012, 116, 1838-1845.

[15] M. A. Blanco, Á. Martín Pendás, E. Francisco, J. Chem. Theory Comput. 2005, 1, 1096-1109.

[16] E. Francisco, Á. Martín Pendás, M. Blanco, J. Chem. Theory Comput. 2006, 2, 90-102.

[17] J. M. Guevara-Vela, R. Chávez-Calvillo, M. García-Revilla, J. Hernández-Trujillo, O. Christiansen, E. Francisco, A. Martín Pendás, T. Rocha-Rinza, Chem. Eur. J. 2013, 19, 14304-15.

[18] J. M. Guevara-Vela, E. Romero-Montalvo, V. A. Mora Gomez, R. Chavez-Calvillo, M. Garcia-Revilla, E. Francisco, A. M. Pendas, T. Rocha-Rinza, Phys. Chem. Chem. Phys. 2016, 18, 19557-19566. 
[19] D. Tiana, E. Francisco, P. Macchi, A. Sironi, A. Martín Pendás, J. Phys. Chem. A 2015, 2, 150209003041008 .

[20] O. A. Syzgantseva, V. Tognetti, L. Joubert, J. Phys. Chem. A 2013, 117, 8969-8980.

[21] M. Yahia-Ouahmed, V. Tognetti, L. Joubert, Comp. Theor. Chem. 2015, 1053, 254-262.

[22] K. Eskandari, M. Lesani, Chem. Eur. J. 2015, 21, 4739-4746.

[23] A. D. Becke, K. E. Edgecombe, J. Chem. Phys. 1990, 92, 5397.

[24] B. Silvi, A. Savin, Nature 1994, 371, 683.

[25] A. Savin, R. Nesper, S. Wengert, T. F. Fässler, Angew. Chem. Int. Ed. 1997, 36, 1808.

[26] Y. Zhao, D. G. Truhlar, Theoretical Chemistry Accounts 2007, 120, 215-241.

[27] T. H. Dunning, J. Chem. Phys. 1989, 90.

[28] M. J. Frisch, G. W. Trucks, H. B. Schlegel, G. E. Scuseria, M. A. Robb, J. R. Cheeseman, G. Scalmani, V. Barone, B. Mennucci, G. A. Petersson, H. Nakatsuji, M. Caricato, X. Li, H. P. Hratchian, A. F. Izmaylov, J. Bloino, G. Zheng, J. L. Sonnenberg, M. Hada, M. Ehara, K. Toyota, R. Fukuda, J. Hasegawa, M. Ishida, T. Nakajima, Y. Honda, O. Kitao, H. Nakai, T. Vreven, J. A. Montgomery, Jr., J. E. Peralta, F. Ogliaro, M. Bearpark, J. J. Heyd, E. Brothers, K. N. Kudin, V. N. Staroverov, R. Kobayashi, J. Normand, K. Raghavachari, A. Rendell, J. C. Burant, S. S. Iyengar, J. Tomasi, M. Cossi, N. Rega, J. M. Millam, M. Klene, J. E. Knox, J. B. Cross, V. Bakken, C. Adamo, J. Jaramillo, R. Gomperts, R. E. Stratmann, O. Yazyev, A. J. Austin, R. Cammi, C. Pomelli, J. W. Ochterski, R. L. Martin, K. Morokuma, V. G. Zakrzewski, G. A. Voth, P. Salvador, J. J. Dannenberg, S. Dapprich, A. D. Daniels, Ö.. Farkas, J. B. Foresman, J. V. Ortiz, J. Cioslowski, D. J. Fox, Gaussian 09 Revision C.1, Gaussian Inc. Wallingford CT 2009 .

[29] T. A. Keith, AIMAll, Version 16.01.09, 2012, TK Gristmill Software, aim.tkgristmill.com.

[30] A. Martín Pendás, E. Francisco, Promolden. A QTAIM/IQA code (unpublished).

[31] P. Maxwell, Á. M. Pendás, P. L. A. Popelier, Phys. Chem. Chem. Phys. 2016, 18, 20986-21000.

[32] A. M. Pendás, E. Francisco, M. A. Blanco, C. Gatti, Chemistry (Weinheim an der Bergstrasse, Germany) 2007, 13, 9362-71. 